\title{
Coronary sinus blood flow and coronary haemodynamic function in children: measurement by the continuous thermodilution method with eoronary sinus cannulation via the femoral vein
}

\author{
Kenji Hamaoka, Zenshiro Onouchi, Yasutaka Kamiya
}

\begin{abstract}
In 19 children with Kawasaki disease without any cardiac sequelae the coronary sinus was cannulated via the femoral vein with a specially designed flow catheter and coronary sinus blood flow was measured by the continuous thermodilution method. There was a statistically significant positive correlation between coronary sinus blood flow and age, body surface area, and left ventricular mass, but coronary sinus blood flow per left ventricular mass $(\mathbf{1 0 0} \mathrm{g})$ was negatively correlated with age, body surface area, and left ventricular mass. Coronary vascular resistance was negatively correlated with age, body surface area, and left ventricular mass.

Younger children require a much greater coronary blood flow per left ventricular mass and have a higher coronary vascular resistance than older children and adolescents. These results may indicate that coronary blood flow is less efficient in childhood than in adolescence or adulthood.
\end{abstract}

The supply of oxygen to the myocardium is determined mainly by the volume of the coronary blood flow. Therefore, measurement of coronary blood flow is a useful way of evaluating coronary lesions and myocardial ischaemia. Measurement of coronary blood flow and the evaluation of the coronary haemodynamic function by arteriography provides important clinical information for the management of children with the coronary sequelae of Kawasaki disease. However, there has been no systematic report on the coronary blood flow or coronary haemodynamic function in childhood because catheterisation of the coronary sinus is difficult in children. We have reported a technique for easier cannulation of the coronary sinus via the femoral vein during routine cardiac catheterisation. ${ }^{1}$ To investigate the characteristics of coronary haemodynamic function in childhood, we measured the coronary sinus blood flow by the continuous thermodilution method using our specially designed flow catheter in children with Kawasaki disease without any sequelae.

\section{Patients and methods}

In the past two years, coronary angiography was carried out in 30 patients to detect the coronary sequelae of Kawasaki disease and to evaluate the coronary haemodynamic function. In the present study we analysed the findings in 19 ( 15 boys and four girls, aged 2 to 18 years (mean 8 )) of the 30 patients who were shown to have normal coronary arteries despite echocardiographic evidence of dilatation. The interval from the onset of Kawasaki disease to the time of this study in the 19 patients ranged from one to 15 years (mean 6.7 years) The nature of the study was discussed with the patients' parents and then written consent was obtained.

After left ventricular angiography and selective coronary angiography, the coronary sinus flow catheter (fig 1A) (Webster Co, CCU-7U-90B), which was designed in our laboratory, was introduced through the femoral vein into the coronary sinus by a technique reported previously ${ }^{1}$ (fig 1B). The coronary sinus flow catheter has an injection orifice at the tip and an external thermistor $12 \mathrm{~mm}$ from the tip. The position of the catheter was confirmed by angiography. In addition, a pigtail catheter was inserted through the femoral artery into the ascending aorta. Coronary sinus blood flow was measured by the continuous thermodilution method $^{2}>30$ minutes after the angiographic studies. The coronary vascular resistance was calculated as the ratio of mean arterial pressure $(\mathrm{mm} \mathrm{Hg}$ ) to coronary sinus blood flow $(\mathrm{ml} / \mathrm{min})$. Left ventricular mass was calculated from the left ventricular angiogram. ${ }^{3}$

\section{Results}

In the 19 patients, coronary sinus blood flow at rest increased significantly with age: age 2-4 years, $51.9(10.4)($ mean $(\mathrm{SD})) \mathrm{ml} / \mathrm{min}$; age 59 years, $75.8(8.7) \mathrm{ml} / \mathrm{min}$; age $10-18$ years, $102.4(17 \cdot 7) \mathrm{ml} / \mathrm{min}$ (fig $2 \mathrm{~A})$. Coronary sinus blood flow was positively correlated with age, body surface area, and left ventricular mass 
Figure 1 (A) Specially designed coronary sinus flow catheters; type 1 was for young and older children and type 2 for adolescents and adults. (B) Coronary sinus flow catheter inserted through the femoral vein into the coronary sinus.

Figure 2 Relations between coronary sinus blood flow (CSBF) and aging $(A)$, body surface area $(B S A)(B)$, and left ventricular $(L V)$ mass (C).
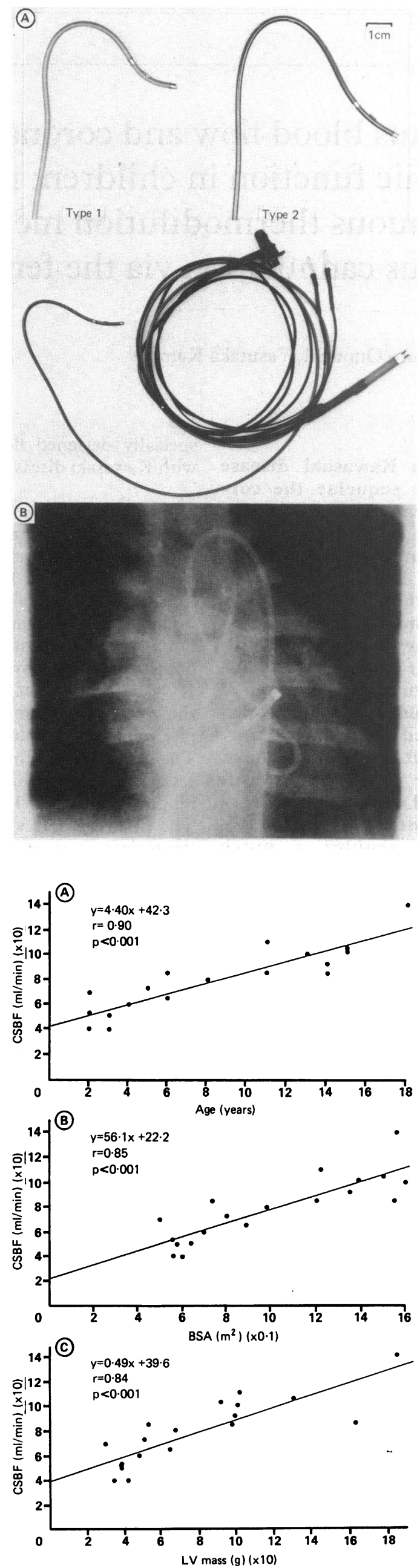

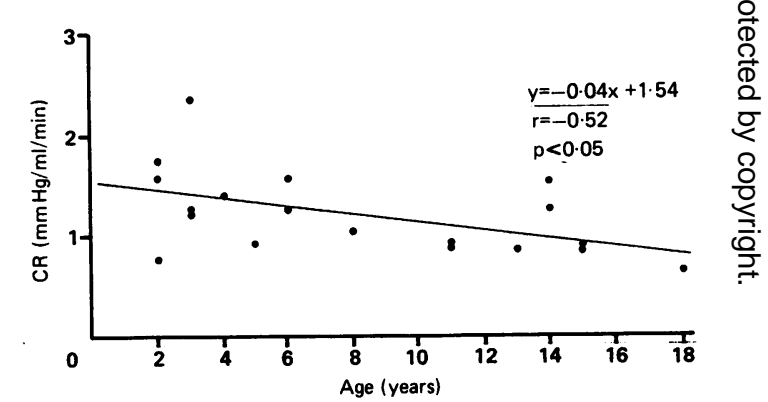

Figure 4 Relation between coronary vascular resistance (CR) and age.
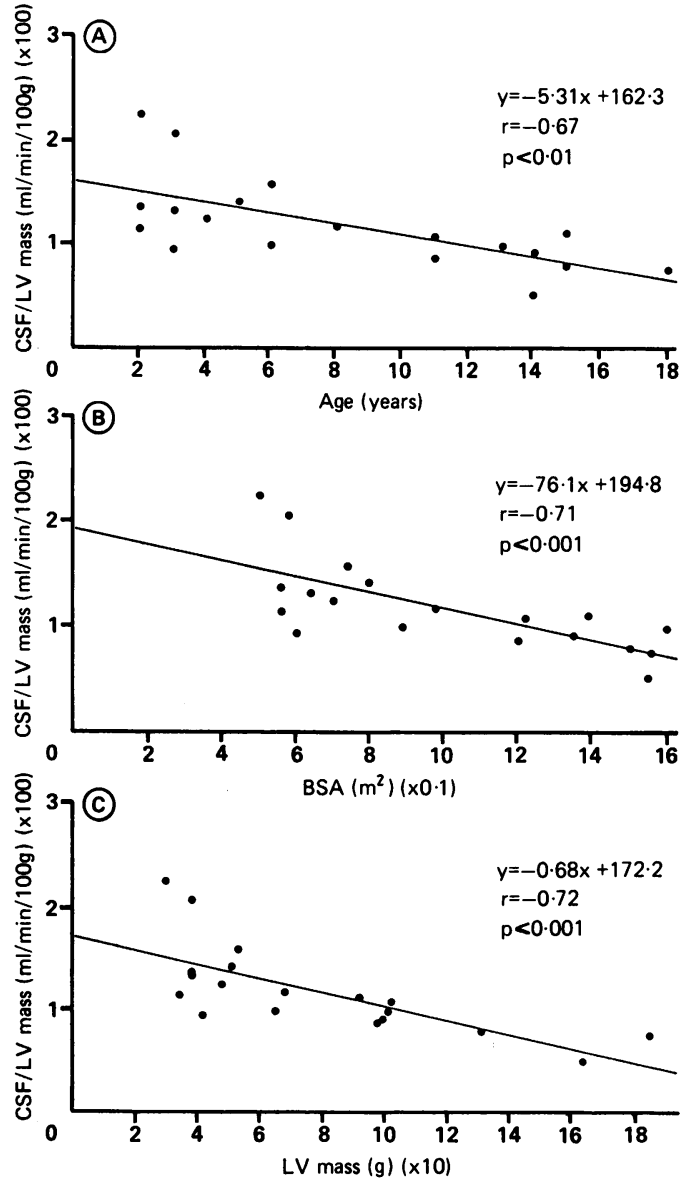

Figure 3 Relations between coronary sinus blood flow per $100 \mathrm{~g}$ left ventricular mass (age) (A), body surface area $(B)$, and left ventricular mass $(C)$

(fig 2). Coronary sinus blood flow per left ventricular mass $(100 \mathrm{~g})$, however, was negatively correlated with age, body surface area, and left ventricular mass (fig 3). Furthermore, in the 19 patients, coronary vascular resistance was negatively correlated with age (fig 4), body surface area ( $\mathrm{r}=-0.55$, $\mathrm{p}<0.02)$, and left ventricular mass $(\mathrm{r}=$ $-0.53, \mathrm{p}<0.02)$. The rate-pressure product did not correlate with age $(r=-0.20$, $\mathrm{p}<0.5)$ or coronary sinus blood flow $(\mathrm{r}=$ $0.22, \mathrm{p}<0.5)$, and the coronary sinus blood flow did not correlate with heart rate $(r=$ $-0.44, \mathrm{p}<0.1)$.

\section{Discussion} flow in childhood was positively correlated with age, body surface area, and left ventricular mass. Coronary sinus blood flow in adults
This study showed that coronary sinus blood 
ranged from 90 to $130 \mathrm{ml} / \mathrm{min}^{24-6}$ Our findings indicate that coronary sinus blood flow in children over 10 years of age was comparable to that in adults. But younger children required a greater coronary sinus blood flow per left ventricular mass than older children or adolescents. Furthermore, the coronary vascular resistance in early childhood was higher than in late childhood and adolescence. These results suggest that coronary blood flow in early childhood was less efficient than in late childhood or adulthood. Therefore, these characteristic coronary flow patterns should be borne in mind during the evaluation of coronary haemodynamic function in childhood.

We thank the following colleagues for participating in this study: Shosei Hayashi, Eikan Koh, Masao Nakagawa,
Toshiyuki Itoi, Isao Shiraishi, Takashi Hayano, Hiroshi Fukumochi, Koichi Sakata.

1 Hamaoka K, Itoi T, Nakagawa M, Kamiya Y, Sawada T. Coronary sinus cannulation via the femoral vein. Pediatr Cardiol 1989;10:91-2.

2 Ganz W, Tamura K, Marcus H, Donoso R, Yoshida S, Swan H. Measurement of coronary sinus blood flow by conH. Measurement of coronary sinus blood flow by con-
tinuous thermodilution in man. Circulation 1964;44: 181-95.

3 Rackly C, Dodge H, Coble Y, Hay R. A method for determining left ventricular mass in man. Circulation

4 Tamura K, Hirano O, Honda T, Muto N, Murooka H. Reproducibility and magnitude of coronary sinus ostial and great cardiac vein flows by continuous local thermodilution method in man. Jpn Circ J 1973;37:917-26.

5 Kodama K, Nanto S. Coronary sinus catheterization. Coronary 1987;4:161-70. [In Japanese.]

6 Mikuniya A, Fukuhara Y, Ko M, et al. Estimation of coronary hemodynamics and myocardial metabolism in spontaneous angina pectoris associated with ST-elevation or depression-with reference to anginal attack by ergonovine. Heart 1985;17:388-98. [In Japanese.] 